\title{
QUANTUM EQUIVALENCE PRINCIPLE
}

\author{
Hagen Kleinert \\ Institut für Theoretische Physik \\ Freie Universität Berlin \\ Arnimallee 14 \\ D-14195 Berlin, Germany
}

A simple mapping procedure is presented by which classical orbits and path integrals for the motion of a point particle in flat space can be transformed directly into those in curved space with torsion. Our procedure evolved from well-established methods in the theory of plastic deformations, where crystals with defects are described mathematically as images of ideal crystals under active nonholonomic coordinate transformations. Our mapping procedure may be viewed as a natural extension of Einstein's famous equivalence principle. When applied to time-sliced path integrals, it gives rise to a new quantum equivalence principle which determines short-time action and measure of fluctuating orbits in spaces with curvature and torsion. The nonholonomic transformations possess a nontrivial Jacobian in the path integral measure which produces in a curved space an additional term proportional to the curvature scalar $R$, thus canceling a similar term found earlier by DeWitt. This cancelation is important for correctly describing semiclassically and quantum mechanically various systems such as the hydrogen atom, a particle on the surface of a sphere, and a spinning top. It is also indispensable for the process of bosonization, by which Fermi particles are redescribed by those fields.

Lectures presented at the 1996 Summer School on Path Integration in Cargèse, Corse.

Email: kleinert@physik.fu-berlin.de;

URL: http://www.physik.fu-berlin.de/ ₹leinert;

Phone/Fax: 0049/30/8383034

Source and postscript available from eprint archive (quant-ph/9612040) 


\section{Introduction}

In 1957, Bryce DeWitt [1] proposed a path integral formula for a point particle in a curved space using a specific generalization of Feynman's time-sliced formula in Cartesian coordinates. Surprisingly, his amplitude turned out to satisfy a Schrödinger equation different from what had previously been considered as correct [2]. In addition to the Laplace-Beltrami operator for the kinetic term, his Hamilton operator contained an extra effective potential proportional to the curvature scalar $R$. At the time of his writing, DeWitt could not think of any argument to outrule the presence of such an extra term.

Since DeWitt's pioneering work, the time-sliced path integral in curved spaces has been reformulated by many people in a variety of ways [3]. The basic problem is the freedom in time slicing the functional integral. Literature offers prepoint, midpoint, and postpoint prescriptions which in the Schrödinger equation correspond to different orderings of the momentum operators $\hat{p}_{\mu}$ with respect to the position variables $q^{\lambda}$ in the Hamiltonian operator $\hat{H}=g^{\mu \nu}(q) \hat{p}_{\mu} \hat{p}_{\nu} / 2$. Similar ambiguities are well known in the theory of stochastic differential equations where different algorithms have been developed by Itô and Stratonovich based on different time discretization procedures [1]. In the stochastic context, covariant versions of the Fokker-Planck equation in curved spaces have been derived by Graham [5]. The mathematical approach to path integrals uses techniques [6] similar to the stochastic one. The inherent ambiguities can be removed by demanding a certain form for the Schrödinger equation of the system, which in curved space has the Laplace-Beltrami operator as an operator for the kinetic energy [2], without an additional curvature scalar.

It has often been repeated that a Hamiltonian whose kinetic term depends on the position variable has in principle many different operator versions. For an arbitrary model Hamiltonian, this is of course, true. A specific physical system, however, must have a unique Hamilton operator. If a system has a high symmetry, it is often possible to find its correct form on the basis of group theory. Recall that in standard textbooks on quantum mechanics [7], a spinning top is quantized by expressing its Hamiltonian in terms of the generators of the rotation group, and quantizing these via the wellknown commutation rules, rather than canonical variables. This procedure avoids the ordering problem by avoiding canonical variables. The resulting Hamilton operator contains only a Laplace-Beltrami operator and no extra term proportional to $R$. A particle on the surface of a sphere is quantized similarly. This procedure forms the basis of the so-called group quantization or geometric quantization [8] which corresponds to Schrödinger equations containing only the Laplace-Beltrami operator and no extra curvature terms.

Until recently, geometric quantization was the only procedure which predicted the form of the Schrödinger equation uniquely on the basis of symmetry, with generally accepted results. Moreover, canonical quantization in flat space is a special case since it corresponds to a geometric quantization of the generators of the euclidean group. Unfortunately, it is quite difficult to generalize this procedure to systems in more general geometries without symmetry. In particular, it makes no prediction as to the form of 
the Schrödinger equation in spaces with curvature and torsion. In DeWitt's time-sliced approach and its various successors, Schrödinger equations are found which contain many possible selections of scalar combinations of curvature and torsion tensor. As a consequence, there is a definite need for a principle capable of predicting the correct Schrödinger equation in such spaces.

In the context of gravity, this may seem a somewhat academic question since nobody has ever experimentally observed a scalar term in the Schrödinger equation of gravitating matter for a point particle in a curved space, even if torsion is neglected, and it is not even clear, whether gravity will generate torsion outside spinning matter. The simplest generalization of Einstein's theory to the Einstein-Cartan theory [9] does not permit propagating torsion.

Fortunately, there exist fields other than gravitational physics and accessible to experiment, where torsion enters geometry. Most notable is the field of defect physics, where geometric methods have been used successfully for a long time to describe the plastic properties of materials [9]. Defects are described mathematically by means of active nonholonomic coordinate transformations.t. They will be described in detail in Section 2. In Fig. 1 we show two typical elementary defects in two dimensions which can be generated by such transformations. It has been understood a long time ago that, geometrically, crystals with defects correspond to spaces with curvature and torsion [10].

In the context of path integrals, such transformations are of crucial importance. They provided us with a key to finding the resolvent of the most elementary atomic system, the hydrogen atom [11]. Two such transformations brought it to a harmonic form. Only recently was it recognized that one of these transformations may be interpreted as leading to a space with torsion [12]. If DeWitt's construction rules for a path integral in curved space would be adapted to this case, the resulting path integral, would produce the wrong atomic spectrum.

The resolution of this puzzle has led to the discovery of a simple rule for correctly transforming Feynman's time-sliced path integral formula from its well-known Cartesian form to spaces with curvature and torsion [12, 13, 114. The rule plays the same fundamental role in quantum physics as Einstein's equivalence principle does within classical physics, where it governs the form of the equations of motion in curved spaces. It is therefore called quantum equivalence principle (QEP) [12].

The crucial place where this principle makes a nontrivial statement is in the measure of the path integral. The nonholonomic nature of the differential coordinate transformation gives rise to an additional term with respect to the naive DeWitt measure, and this cancels precisely the bothersome term proportional to $R$ found by DeWitt.

It is the purpose of these lectures to demonstrate the power of the new quantum equivalence principle and to discuss its consequences also at the classical level, where the familiar action principle breaks down and requires an important modification [12, 15, 16. The geometric reason for this lies in the fact that infinitesimal variations can

\footnotetext{
${ }^{1}$ Note that passive nonholonomic coordinate transformations lead to an alternative, usually inconvenient description of ideal crystals. The role of torsion is then played by so-called objects of anholonomity.
} 
no longer be taken as closed curves; they possess a defect analogous to the Burgers vector in crystal physics. This surprising result has been verified by deriving the Euler equations for the motion of a spinning top from an action principle formulated within the body-fixed reference frame, where the geometry of the nonholonomic coordinates possesses torsion [18.

\section{Classical Motion of a Mass Point in a Space with Torsion}

We begin by recalling that Einstein formulated the rules for finding the classical laws of motion in a gravitational field on the basis of his famous equivalence principle. He assumed the space to be free of torsion since otherwise his geometric principle was not able to determine the classical equations of motion uniquely. Since our nonholonomic mapping principle is free of this problem, we do not need to restrict the geometry in this way. The correctness of the resulting laws of motion is exemplified by several physical systems with well-known experimental properties. Basis for these "experimental verifications" will be the fact that classical equations of motion are invariant under nonholonomic coordinate transformations. Since it is well known that active versions of such transformations introduce curvature and torsion into a parameter space, such redescriptions of standard mechanical systems provide us with sample systems in general metric-affine spaces.

To be as specific and as simple as possible, we restrict ourselves to the theory for a nonrelativistic massive point particle in a general metric-affine space. The entire discussion may easily be extended to relativistic particles in spacetime.

\subsection{Equations of Motion}

Consider the action of the particle along the orbit $\mathbf{x}(t)$ in a flat space parametrized with $D$ rectilinear, Cartesian coordinates:

$$
\mathcal{A}=\int_{t_{a}}^{t_{b}} d t \frac{M}{2}\left(\dot{x}^{i}\right)^{2}
$$

It may be transformed to curvilinear coordinates $q^{\mu}, \mu=1,2,3$, via some functions

$$
x^{i}=x^{i}(q)
$$

leading to

$$
\mathcal{A}=\int_{t_{a}}^{t_{b}} d t \frac{M}{2} g_{\mu \nu}(q) \dot{q}^{\mu} \dot{q}^{\nu}
$$

where

$$
g_{\mu \nu}(q)=\partial_{\mu} x^{i}(q) \partial_{\nu} x^{i}(q)
$$

is the induced metric for the curvilinear coordinates. Repeated indices are understood to be summed over, as usual. For Cartesian coordinates, upper and lower indices $i$ are 
the same. The indices $\mu, \nu$ of the curvilinear coordinates, on the other hand, are lowered by contraction with the metric $g_{\mu \nu}$ or raised with the inverse metric $g^{\mu \nu} \equiv\left(g_{\mu \nu}\right)^{-1}$.

The length of the orbit in the flat space is given by

$$
l=\int_{t_{a}}^{t_{b}} d t \sqrt{g_{\mu \nu}(q) \dot{q}^{\mu} \dot{q}^{\nu}}
$$

Both the action (3) and the length (5) are invariant under arbitrary reparametrizations of space $q^{\mu} \rightarrow q^{\prime \mu}$.

Einstein's equivalence principle amounts to the postulate that the transformed action (3) describes directly the motion of the particle in the presence of a gravitational field caused by other masses. The forces caused by the field are all a result of the geometric properties of the metric tensor.

The equations of motion are obtained by extremizing the action in Eq. (3) with the result

$$
\partial_{t}\left(g_{\mu \nu} \dot{q}^{\nu}\right)-\frac{1}{2} \partial_{\mu} g_{\lambda \nu} \dot{q}^{\lambda} \dot{q}^{\nu}=g_{\mu \nu} \ddot{q}^{\nu}+\bar{\Gamma}_{\lambda \nu \mu} \dot{q}^{\lambda} \dot{q}^{\nu}=0
$$

Here

$$
\bar{\Gamma}_{\lambda \nu \mu} \equiv \frac{1}{2}\left(\partial_{\lambda} g_{\nu \mu}+\partial_{\nu} g_{\lambda \mu}-\partial_{\mu} g_{\lambda \nu}\right)
$$

is the Riemann connection or Christoffel symbol of the first kind. Defining also the Christoffel symbol of the second kind

$$
\bar{\Gamma}_{\lambda \nu}^{\mu} \equiv g^{\mu \sigma} \bar{\Gamma}_{\lambda \nu \sigma}
$$

we can write

$$
\ddot{q}^{\mu}+\bar{\Gamma}_{\lambda \nu}{ }^{\mu} \dot{q}^{\lambda} \dot{q}^{\nu}=0 .
$$

The solutions of these equations are the classical orbits. They coincide with the extrema of the length $l$ of a curve in (5). Thus, in a curved space, classical orbits are the shortest curves, called geodesics. The reason for the name shortest lines is that they minimize the invariant length (5) of all lines connecting two given points $q_{a}^{\mu}=q^{\mu}\left(t_{a}\right)$ and $q_{b}^{\mu}=q^{\mu}\left(t_{b}\right)$.

The same equations can also be obtained directly by transforming the equation of motion from

$$
\ddot{x}^{i}=0
$$

to curvilinear coordinates $q^{\mu}$, which gives

$$
\ddot{x}^{i}=\frac{\partial x^{i}}{\partial q^{\mu}} \ddot{q}^{\mu}+\frac{\partial^{2} x^{i}}{\partial q^{\lambda} \partial q^{\nu}} \dot{q}^{\lambda} \dot{q}^{\nu}=0 .
$$

At this place it is useful to employ the so-called basis triads

$$
e_{\mu}^{i}(q) \equiv \frac{\partial x^{i}}{\partial q^{\mu}}
$$


and the reciprocal basis triads

$$
e_{i}{ }^{\mu}(q) \equiv \frac{\partial q^{\mu}}{\partial x^{i}}
$$

which satisfy the orthogonality and completeness relations

$$
\begin{aligned}
& e_{i}{ }^{\mu} e^{i}{ }_{\nu}=\delta^{\mu}{ }_{\nu}, \\
& e_{i}{ }^{\mu} e^{j}{ }_{\mu}=\delta_{i}{ }^{j} .
\end{aligned}
$$

The induced metric can then be written as

$$
g_{\mu \nu}(q)=e_{\mu}^{i}(q) e_{\nu}^{i}(q) .
$$

Using the basis triads, Eq. (11) can be rewritten as

$$
\frac{d}{d t}\left(e_{\mu}^{i} \dot{q}^{\mu}\right)=e_{\mu}^{i} \ddot{q}^{\mu}+e_{\mu, \nu}^{i} \dot{q}^{\mu} \dot{q}^{\nu}=0,
$$

or as

$$
\ddot{q}^{\mu}+e_{i}{ }^{\mu} e^{i}{ }_{\kappa, \lambda} \dot{q}^{\kappa} \dot{q}^{\lambda}=0 .
$$

The subscript $\lambda$ separated by a comma denotes the partial derivative $\partial_{\lambda}=\partial / \partial q^{\lambda}$, i.e., $f_{, \lambda} \equiv \partial_{\lambda} f$. The quantity in front of $\dot{q}^{\kappa} \dot{q}^{\lambda}$ is called the affine connection:

$$
\Gamma_{\lambda \kappa}^{\mu}=e_{i}^{\mu} e_{\kappa, \lambda}^{i}
$$

Due to (14), it can also be written as

$$
\Gamma_{\lambda \kappa}{ }^{\mu}=-e^{i}{ }_{\kappa} e_{i}{ }^{\mu}{ }_{\lambda} .
$$

Thus we arrive at the transformed flat-space equation of motion

$$
\ddot{q}^{\mu}+\Gamma_{\kappa \lambda}^{\mu} \dot{q}^{\kappa} \dot{q}^{\lambda}=0 .
$$

The solutions of this equation are called the straightest lines or autoparallels.

If the coordinate transformation $x^{i}(q)$ is smooth and single-valued, it is integrable. This property is expressed by Schwarz's integrability condition, according to which derivatives in front of such a function $x^{i}(q)$ commute:

$$
\left(\partial_{\lambda} \partial_{\kappa}-\partial_{\kappa} \partial_{\lambda}\right) x^{i}(q)=0
$$

Then the triads satisfy the identity

$$
e_{\kappa, \lambda}^{i}=e_{\lambda, \kappa}^{i}
$$

implying that the connection $\Gamma_{\mu \nu}^{\lambda}$ is symmetric in the lower indices. In this case it coincides with the Riemann connection, the Christoffel symbol $\bar{\Gamma}_{\mu \nu}{ }^{\lambda}$. This follows immediately after inserting $g_{\mu \nu}(q)=e^{i}{ }_{\mu}(q) e_{\nu}^{i}(q)$ into (7) and working out all derivatives using (23). Thus, for a space with curvilinear coordinates $q^{\mu}$ which can be reached by an integrable coordinate transformation from a flat space, the autoparallels coincide with the geodesics. 


\subsection{Nonholonomic Mapping to Spaces with Torsion}

It is possible to map the $x^{i}$-space locally into a $q$-space via an infinitesimal transformation

$$
d x^{i}=e_{\mu}^{i}(q) d q^{\mu}
$$

with coefficient functions $e^{i}{ }_{\mu}(q)$ which are not integrable in the sense of Eq. (22), i.e.,

$$
\partial_{\mu} e_{\nu}^{i}(q)-\partial_{\nu} e_{\mu}^{i}(q) \neq 0
$$

Such a mapping will be called nonholonomic. There exists no single-valued function $x^{i}(q)$ for which $e^{i}{ }_{\mu}(q)=\partial x^{i}(q) / \partial q^{\mu}$. Nevertheless, we shall write (25) in analogy to (22) as

$$
\left(\partial_{\lambda} \partial_{\kappa}-\partial_{\kappa} \partial_{\lambda}\right) x^{i}(q) \neq 0
$$

since this equation involves only the differential $d x^{i}$. This violation of mathematical conventions will not cause any problems.

From Eq. 25 we see that the image space of a nonholonomic mapping carries torsion. The connection $\Gamma_{\lambda \kappa}{ }^{\mu}=e_{i}{ }^{\mu} e^{i}{ }_{\kappa, \lambda}$ has a nonzero antisymmetric part, called the torsion tensor [17]:

$$
S_{\lambda \kappa}{ }^{\mu}=\frac{1}{2}\left(\Gamma_{\lambda \kappa}{ }^{\mu}-\Gamma_{\kappa \lambda}{ }^{\mu}\right)
$$

In contrast to $\Gamma_{\lambda \kappa}{ }^{\mu}$, the antisymmetric part $S_{\lambda \kappa}{ }^{\mu}$ is a proper tensor under holonomic coordinate transformations. The contracted tensor

$$
S_{\mu} \equiv S_{\mu \lambda}^{\lambda}
$$

transforms like a vector, whereas the contracted connection $\Gamma_{\mu} \equiv \Gamma_{\mu \nu}{ }^{\nu}$ does not. Even though $\Gamma_{\mu \nu}{ }^{\lambda}$ is not a tensor, we shall freely lower and raise its indices using contractions with the metric or the inverse metric, respectively: $\Gamma^{\mu}{ }_{\nu}^{\lambda} \equiv g^{\mu \kappa} \Gamma_{\kappa \nu}{ }^{\lambda}, \Gamma_{\mu}{ }^{\nu \lambda} \equiv g^{\nu \kappa} \Gamma_{\mu \kappa}{ }^{\lambda}$, $\Gamma_{\mu \nu \lambda} \equiv g_{\lambda \kappa} \Gamma_{\mu \nu}{ }^{\kappa}$. The same thing will be done with $\bar{\Gamma}_{\mu \nu}{ }^{\lambda}$.

In the presence of torsion, the connection is no longer equal to the Christoffel symbol. In fact, by rewriting $\Gamma_{\mu \nu \lambda}=e_{i \lambda} \partial_{\mu} e^{i}{ }_{\nu}$ trivially as

$$
\begin{gathered}
\Gamma_{\mu \nu \lambda}=\frac{1}{2}\left\{e_{i \lambda} \partial_{\mu} e^{i}{ }_{\nu}+\partial_{\mu} e_{i \lambda} e^{i}{ }_{\nu}+e_{i \mu} \partial_{\nu} e_{\lambda}^{i}+\partial_{\nu} e_{i \mu} e_{\lambda}^{i}-e_{i \mu} \partial_{\lambda} e^{i}{ }_{\nu}-\partial_{\lambda} e_{i \mu} e^{i}{ }_{\nu}\right\} \\
+\frac{1}{2}\left\{\left[e_{i \lambda} \partial_{\mu} e_{\nu}^{i}-e_{i \lambda} \partial_{\nu} e^{i}{ }_{\mu}\right]-\left[e_{i \mu} \partial_{\nu} e^{i}{ }_{\lambda}-e_{i \mu} \partial_{\lambda} e^{i}{ }_{\nu}\right]+\left[e_{i \nu} \partial_{\lambda} e^{i}{ }_{\mu}-e_{i \nu} \partial_{\mu} e^{i}{ }_{\lambda}\right]\right\}
\end{gathered}
$$

and using $e_{\mu}^{i}(q) e_{\nu}^{i}(q)=g_{\mu \nu}(q)$, we find the decomposition

$$
\Gamma_{\mu \nu}^{\lambda}=\bar{\Gamma}_{\mu \nu}^{\lambda}+K_{\mu \nu}^{\lambda}
$$

where the combination of torsion tensors

$$
K_{\mu \nu \lambda} \equiv S_{\mu \nu \lambda}-S_{\nu \lambda \mu}+S_{\lambda \mu \nu}
$$

is called the contortion tensor. It is antisymmetric in the last two indices so that

$$
\Gamma_{\mu \nu}{ }^{\nu}=\bar{\Gamma}_{\mu \nu}{ }^{\nu}
$$


In Einstein's theory of gravitation, torsion is assumed to be absent, i.e., the integrability condition for $x^{i}(q)$ is not violated as in (26). The main effect of matter in Einstein's theory of gravitation manifests itself in the violation of the integrability condition for the derivative of the coordinate transformation $x^{i}(q)$, namely,

$$
\left(\partial_{\mu} \partial_{\nu}-\partial_{\nu} \partial_{\mu}\right) \partial_{\lambda} x^{i}(q) \neq 0
$$

A transformation for which $x^{i}(q)$ itself is integrable, while the first derivatives $\partial_{\mu} x^{i}(q)=$ $e^{i}{ }_{\mu}(q)$ are not, carries a flat-space region into a purely curved one. The quantity which records the nonintegrability is the Cartan curvature tensor

$$
R_{\mu \nu \lambda}^{\kappa}=e_{i}^{\kappa}\left(\partial_{\mu} \partial_{\nu}-\partial_{\nu} \partial_{\mu}\right) e_{\lambda}^{i} .
$$

Working out the derivatives using (19) we see that $R_{\mu \nu \lambda}{ }^{\kappa}$ can be written as a covariant curl of the connection,

$$
R_{\mu \nu \lambda}{ }^{\kappa}=\partial_{\mu} \Gamma_{\nu \lambda}{ }^{\kappa}-\partial_{\nu} \Gamma_{\mu \lambda}{ }^{\kappa}-\left[\Gamma_{\mu}, \Gamma_{\nu}\right]_{\lambda}{ }^{\kappa} .
$$

In the last term we have used a matrix notation for the connection. The tensor components $\Gamma_{\mu \lambda}{ }^{\kappa}$ are viewed as matrix elements $\left(\Gamma_{\mu}\right)_{\lambda}{ }^{\kappa}$, so that we can use the matrix commutator

$$
\left[\Gamma_{\mu}, \Gamma_{\nu}\right]_{\lambda}{ }^{\kappa} \equiv\left(\Gamma_{\mu} \Gamma_{\nu}-\Gamma_{\nu} \Gamma_{\mu}\right)_{\lambda}{ }^{\kappa}=\Gamma_{\mu \lambda}{ }^{\sigma} \Gamma_{\nu \sigma}{ }^{\kappa}-\Gamma_{\nu \lambda}{ }^{\sigma} \Gamma_{\mu \sigma}{ }^{\kappa}
$$

Einstein's original theory of gravity assumes the absence of torsion. The space properties are completely specified by the Riemann curvature tensor formed from the Riemann connection (the Christoffel symbol)

$$
\bar{R}_{\mu \nu \lambda}{ }^{\kappa}=\partial_{\mu} \bar{\Gamma}_{\nu \lambda}{ }^{\kappa}-\partial_{\nu} \bar{\Gamma}_{\mu \lambda}{ }^{\kappa}-\left[\bar{\Gamma}_{\mu}, \bar{\Gamma}_{\nu}\right]_{\lambda}{ }^{\kappa} .
$$

The relation between the two curvature tensors is

$$
R_{\mu \nu \lambda}{ }^{\kappa}=\bar{R}_{\mu \nu \lambda}{ }^{\kappa}+\bar{D}_{\mu} K_{\nu \lambda}{ }^{\kappa}-\bar{D}_{\nu} K_{\mu \lambda}{ }^{\kappa}-\left[K_{\mu}, K_{\nu}\right]_{\lambda}{ }^{\kappa} .
$$

In the last term, the $K_{\mu \lambda}{ }^{\kappa}$ 's are viewed as matrices $\left(K_{\mu}\right)_{\lambda}{ }^{\kappa}$. The symbols $\bar{D}_{\mu}$ denote the covariant derivatives formed with the Christoffel symbol. Covariant derivatives act like ordinary derivatives if they are applied to a scalar field. When applied to a vector field, they act as follows:

$$
\begin{aligned}
\bar{D}_{\mu} v_{\nu} & \equiv \partial_{\mu} v_{\nu}-\bar{\Gamma}_{\mu \nu}{ }^{\lambda} v_{\lambda}, \\
\bar{D}_{\mu} v^{\nu} & \equiv \partial_{\mu} v^{\nu}+\bar{\Gamma}_{\mu \lambda}{ }^{\nu} v^{\lambda} .
\end{aligned}
$$

The effect upon a tensor field is the generalization of this; every index receives a corresponding additive $\bar{\Gamma}$ contribution.

In the presence of torsion, there exists another covariant derivative formed with the affine connection $\Gamma_{\mu \nu}{ }^{\lambda}$ rather than the Christoffel symbol which acts upon a vector field as

$$
\begin{aligned}
D_{\mu} v_{\nu} & \equiv \partial_{\mu} v_{\nu}-\Gamma_{\mu \nu}{ }^{\lambda} v_{\lambda} \\
D_{\mu} v^{\nu} & \equiv \partial_{\mu} v^{\nu}+\Gamma_{\mu \lambda}{ }^{\nu} v^{\lambda}
\end{aligned}
$$


The two derivatives (38) and (39) are equally covariant under holonomic coordinate transformations. Thus, in conventional differential geometry it is not clear which of them should play a more fundamental role in physics. They do differ, however, in their transformation behavior under nonholonomic transformations, and there (39) is definitely the preferred object for reasons of simplicity.

From either of the two curvature tensors, $R_{\mu \nu \lambda}{ }^{\kappa}$ and $\bar{R}_{\mu \nu \lambda}{ }^{\kappa}$, one can form the oncecontracted tensors of rank 2, the Ricci tensor

$$
R_{\nu \lambda}=R_{\mu \nu \lambda}^{\mu}
$$

and the curvature scalar

$$
R=g^{\nu \lambda} R_{\nu \lambda} .
$$

The celebrated Einstein equation for the gravitational field postulates that the tensor

$$
G_{\mu \nu} \equiv R_{\mu \nu}-\frac{1}{2} g_{\mu \nu} R,
$$

the so-called Einstein tensor, is proportional to symmetric energy-mo-mentum tensor of all matter fields. This postulate was made only for spaces with no torsion, in which case $R_{\mu \nu}=\bar{R}_{\mu \nu}$ and $R_{\mu \nu}, G_{\mu \nu}$ are both symmetric. As mentioned in the Introduction, it is not yet clear how Einstein's field equations should be generalized in the presence of torsion since the experimental consequences are as yet too small to be observed. In this paper, we are not concerned with the generation of curvature and torsion but only with their consequences upon the motion of point particles.

\subsection{Simple Nonholonomic Sample Mappings}

The generation of defects illustrated in Fig. 1 provides us with two simple examples for nonholonomic mappings which show us in which way these mappings are capable of generating a space with curvature and torsion from a euclidean space. The reader not familiar with this subject is advised to consult the standard literature on this subject quoted in Ref. [9, 10].

Consider first the upper example in Fig. 1, in which a dislocation is generated, characterized by a missing or an additional layer of atoms. In two dimensions, it may be described differentially by the transformation

$$
d x^{i}= \begin{cases}d q^{1} & \text { for } i=1, \\ d q^{2}+\varepsilon \partial_{\mu} \phi(q) d q^{\mu} & \text { for } i=2,\end{cases}
$$

with infinitesimal $\epsilon$ and the multi-valued function

$$
\phi(q) \equiv \arctan \left(q^{2} / q^{1}\right) .
$$

The triads reduce to dyads, with the components

$$
\begin{aligned}
& e^{1}{ }_{\mu}=\delta^{1}{ }_{\mu}, \\
& e^{2}{ }_{\mu}=\delta^{2}{ }_{\mu}+\epsilon \partial_{\mu} \phi(q),
\end{aligned}
$$


and the torsion tensor has the components

$$
e_{\lambda}^{1} S_{\mu \nu}^{\lambda}=0, \quad e_{\lambda}^{2} S_{\mu \nu}^{\lambda}=\frac{\epsilon}{2}\left(\partial_{\mu} \partial_{\nu}-\partial_{\nu} \partial_{\mu}\right) \phi
$$

If we differentiate (44) formally, we find $\left(\partial_{\mu} \partial_{\nu}-\partial_{\nu} \partial_{\mu}\right) \phi \equiv 0$. This, however, is incorrect at the origin. Using Stokes' theorem we see that

$$
\int d^{2} q\left(\partial_{1} \partial_{2}-\partial_{2} \partial_{1}\right) \phi=\oint d q^{\mu} \partial_{\mu} \phi=\oint d \phi=2 \pi
$$

for any closed circuit around the origin, implying that there is a $\delta$-function singularity at the origin with

$$
e_{\lambda}^{2} S_{12}^{\lambda}=\frac{\epsilon}{2} 2 \pi \delta^{(2)}(q) \text {. }
$$

By a linear superposition of such mappings we can generate an arbitrary torsion in the $q$-space. The mapping introduces no curvature. When encircling a dislocation along a closed path $C$, its counter image $C^{\prime}$ in the ideal crystal does not form a closed path. The closure failure is called the Burgers vector

$$
b^{i} \equiv \oint_{C^{\prime}} d x^{i}=\oint_{C} d q^{\mu} e^{i}
$$

It specifies the direction and thickness of the layer of additional atoms. With the help of Stokes' theorem, it is seen to measure the torsion contained in any surface $S$ spanned by $C$ :

$$
b^{i}=\oint_{S} d^{2} s^{\mu \nu} \partial_{\mu} e_{\nu}^{i}=\oint_{S} d^{2} s^{\mu \nu} e_{\lambda}^{i} S_{\mu \nu}{ }^{\lambda}
$$

where $d^{2} s^{\mu \nu}=-d^{2} s^{\nu \mu}$ is the projection of an oriented infinitesimal area element onto the plane $\mu \nu$. The above example has the Burgers vector

$$
b^{i}=(0, \epsilon)
$$

A corresponding closure failure appears when mapping a closed contour $C$ in the ideal crystal into a crystal containing a dislocation. This defines a Burgers vector:

$$
b^{\mu} \equiv \oint_{C^{\prime}} d q^{\mu}=\oint_{C} d x^{i} e_{i}^{\mu}
$$

By Stokes' theorem, this becomes a surface integral

$$
\begin{aligned}
b^{\mu} & =\oint_{S} d^{2} s^{i j} \partial_{i} e_{j}{ }^{\mu}=\oint_{S} d^{2} s^{i j} e_{i}{ }^{\nu} \partial_{\nu} e_{j}{ }^{\mu} \\
& =-\oint_{S} d^{2} s^{i j} e_{i}{ }^{\nu} e_{j}{ }^{\lambda} S_{\nu \lambda}{ }^{\mu}
\end{aligned}
$$

the last step following from (20).

The second example is the nonholonomic mapping in the lower part of Fig. 1 generating a disclination which corresponds to an entire section of angle $\Omega$ missing in 
an ideal atomic array. For an infinitesimal angel $\Omega$, this may be described, in two dimensions, by the differential mapping

$$
x^{i}=\delta_{\mu}^{i}\left[q^{\mu}+\Omega \epsilon_{\nu}^{\mu} q^{\nu} \phi(q)\right],
$$

with the multi-valued function (44). The symbol $\epsilon_{\mu \nu}$ denotes the antisymmetric LeviCivita tensor. The transformed metric

$$
g_{\mu \nu}=\delta_{\mu \nu}-\frac{2 \Omega}{q^{\sigma} q_{\sigma}} \epsilon_{\mu \lambda} \epsilon_{\nu \kappa} q^{\lambda} q^{\kappa}
$$

is single-valued and has commuting derivatives. The torsion tensor vanishes since $\left(\partial_{1} \partial_{2}-\partial_{2} \partial_{1}\right) x^{1,2}$ is proportional to $q^{2,1} \delta^{(2)}(q)=0$. The local rotation field $\omega(q) \equiv$ $\frac{1}{2}\left[\partial_{1} x^{2}(q)-\partial_{2} x^{1}(q)\right]$, on the other hand, is equal to the multi-valued function $-\Omega \phi(q)$, thus having the noncommuting derivatives:

$$
\left(\partial_{1} \partial_{2}-\partial_{2} \partial_{1}\right) \omega(q)=-2 \pi \Omega \delta^{(2)}(q)
$$

To lowest order in $\Omega$, this determines the curvature tensor, which in two dimensions posses only one independent component, for instance $R_{1212}$. Using the fact that $g_{\mu \nu}$ has commuting derivatives, $R_{1212}$ can be written as

$$
R_{1212}=\left(\partial_{1} \partial_{2}-\partial_{2} \partial_{1}\right) \omega(q)
$$

\subsection{Straightest versus Shortest Particle Trajectories}

We have seen in Eqs. (38) and (39) that there exist two different types of covariant derivatives. Thus there are two types of parallel vector fields, $v_{\mathrm{a}}^{\mu}$ and $v_{\mathrm{g}}^{\mu}$, defined by

$$
D_{\nu} v_{\mathrm{a}}^{\mu}(q)=0, \quad \bar{D}_{\nu} v_{\mathrm{g}}^{\mu}(q)=0 .
$$

The stream lines of these vector fields are found by introducing an arbitrary parameter $s$ and searching for a function $q^{\mu}(s)$ whose tangent is given by these vector fields:

$$
\frac{d q_{\mathrm{a}}^{\mu}(q)}{d s}=v_{\mathrm{a}}^{\mu}(q), \quad \frac{d q_{\mathrm{g}}^{\mu}(q)}{d s}=v_{\mathrm{g}}^{\mu}(q)
$$

By forming one more derivative with respect to $s$, we find the differential equations for these stream lines

$$
\begin{aligned}
& \ddot{q}_{\mathrm{a}}^{\mu}+\Gamma_{\kappa \lambda}{ }^{\mu} \dot{q}_{\mathrm{a}}^{\kappa} \dot{q}_{\mathrm{a}}^{\lambda}=0, \\
& \ddot{q}_{\mathrm{g}}^{\mu}+\bar{\Gamma}_{\kappa \lambda}{ }^{\mu} \dot{q}_{\mathrm{g}}^{\kappa} \dot{q}_{\mathrm{g}}^{\lambda}=0 .
\end{aligned}
$$

The first are the autoparallels (21), the second are the shortest lines or geodesics. In the presence of torsion, the shortest and straightest lines are no longer equal. This keeps surprising people, since by (27), torsion is the asymmtric part of the connection and the asymmetric part of $\Gamma_{\kappa \lambda}{ }^{\mu}$ certainly drops out of the equation of motion (60). However, 
the decomposition (29) with the contortion tensor (30) shows, that $\Gamma_{\kappa \lambda}{ }^{\mu}$ contains a contribution from torsion also in its symmetric part:

$$
\Gamma_{\{\kappa \lambda\}}^{\mu}=\bar{\Gamma}_{\kappa \lambda}^{\mu}+2 S_{\kappa \lambda}^{\mu}
$$

Since the two types of lines play geometrically an equally favored role, the question arises as to which of them describes the correct classical particle orbits. The answer will be given in the rest of these lectures. Both types of curves are a priori equally good candidates for particle trajectories in a theory of gravitation in which all particles move along geometrically determined paths.

From our nonholonomic mapping principle, a free-particle trajectory in Euclidean space is mapped into the autoparallel. Since we know that, in classical mechanics, equations of motion remain correct under nonholonomic coordinate transformations, we conclude that nature must have chosen the autoparallels as the geometrically distinguished curves along which particles move.

However, this conclusion might be too hasty. The fundamental Hamilton principle of classical mechanics states that particle trajectories should emerge from a variational approach, in which an action $\mathcal{A}[q]$ which is a functional of arbitrary possible paths $q^{\mu}(t)$ is minimized with respect to small changes $\delta q^{\mu}(t)$. If we take as an action the nonholonomic image (3) of the flat-space action (1), and minimize this without varying the endpoints, i.e. with the boundary conditions

$$
\delta q^{\mu}\left(t_{a}\right)=\delta q^{\mu}\left(t_{b}\right)=0
$$

we find for the particle trajectories the geodesic differential equations (61), rather than the autoparallel ones (60).

Which conclusion is physically correct? At first sight, the nonholonomic mapping principle seems to be inconsistent. In Section 2.5 we shall see that consistency can be ensured by a proper extension of Hamilton's principle to particles in spaces with torsion.

\subsection{Classical Action Principle for Spaces with Curvature and Torsion}

We have seen in the last section that for a unique consistent theory in spaces with torsion we must reexamine the Hamilton action principle for the classical motion of a spinless point particle. We must make sure that autoparallels emerge as the extremals of an action (3) that involves only the metric tensor $g_{\mu \nu}$. The action is independent of the torsion and carries only information on the Riemann part of the space geometry. Torsion can therefore enter the equations of motion only via some novel feature of the variation procedure. Since we know how to perform variations of an action in the euclidean $x^{i}$-space, we deduce the correct procedure in the general metric-affine space by transferring the variations $\delta x^{i}(t)$ under the nonholonomic mapping

$$
\dot{q}^{\mu}=e_{i}^{\mu}(q) \dot{x}^{i}
$$


into the $q^{\mu}$-space. Their images are quite different from ordinary variations as illustrated in Fig. 2(a). The variations of the Cartesian coordinates $\delta x^{i}(t)$ are done at fixed end points of the paths. Thus they form closed paths in the $x$-space. Their images, however, lie in a space with defects and thus possess a closure failure indicating the amount of torsion introduced by the mapping. This property will be emphasized by writing the images $\delta q^{\mu}(t)$ and calling them nonholonomic variations.

Let us calculate them explicitly. The paths in the two spaces are related by the integral equation

$$
q^{\mu}(t)=q^{\mu}\left(t_{a}\right)+\int_{t_{a}}^{t} d t^{\prime} e_{i}^{\mu}\left(q\left(t^{\prime}\right)\right) \dot{x}^{i}\left(t^{\prime}\right)
$$

For two neighboring paths in $x$-space differing from each other by a variation $\delta x^{i}(t)$, Eq. (65) determines the nonholonomic variation $\delta q^{\mu}(t)$ :

$$
\delta q^{\mu}(t)=\int_{t_{a}}^{t} d t^{\prime} \delta\left[e_{i}^{\mu}\left(q\left(t^{\prime}\right)\right) \dot{x}^{i}\left(t^{\prime}\right)\right]
$$

A comparison with (64) shows that the variations $\delta q^{\mu}$ and the time derivative of $q^{\mu}$ are independent of each other

$$
\delta \dot{q}^{\mu}(t)=\frac{d}{d t} \delta q^{\mu}(t)
$$

just as for ordinary variations $\delta x^{i}$.

Let us introduce auxiliary holonomic variations in $q$-space:

$$
\delta q^{\mu} \equiv e_{i}^{\mu}(q) \delta x^{i}
$$

In contrast to $\delta q^{\mu}(t)$, these vanish at the endpoints,

$$
\delta q\left(t_{a}\right)=\delta q\left(t_{b}\right)=0,
$$

i.e., they form closed paths with the unvaried orbits.

Using (68) we derive from (66) the relation

$$
\begin{aligned}
\frac{d}{d t} \delta q^{\mu}(t) & =\delta e_{i}{ }^{\mu}(q(t)) \dot{x}^{i}(t)+e_{i}{ }^{\mu}(q(t)) \delta \dot{x}^{i}(t) \\
& =\delta e_{i}{ }^{\mu}(q(t)) \dot{x}^{i}(t)+e_{i}{ }^{\mu}(q(t)) \frac{d}{d t}\left[e^{i}{ }_{\nu}(t) \delta q^{\nu}(t)\right]
\end{aligned}
$$

After inserting

$$
\delta e_{i}^{\mu}(q)=-\Gamma_{\lambda \nu}{ }^{\mu} \delta q^{\lambda} e_{i}^{\nu}, \quad \frac{d}{d t} e^{i}{ }_{\nu}(q)=\Gamma_{\lambda \nu}{ }^{\mu} \dot{q}^{\lambda} e^{i}{ }_{\mu}
$$

this becomes

$$
\frac{d}{d t} \delta q^{\mu}(t)=-\Gamma_{\lambda \nu}^{\mu} \delta q^{\lambda} \dot{q}^{\nu}+\Gamma_{\lambda \nu}^{\mu} \dot{q}^{\lambda} \delta q^{\nu}+\frac{d}{d t} \delta q^{\mu}
$$

It is useful to introduce the difference between the nonholonomic variation $\delta q^{\mu}$ and the auxiliary holonomic variation $\delta q^{\mu}$ :

$$
\delta b^{\mu} \equiv \delta q^{\mu}-\delta q^{\mu}
$$


Then we can rewrite (72) as a first-order differential equation for $\delta b^{\mu}$ :

$$
\frac{d}{d t} \delta b^{\mu}=-\Gamma_{\lambda \nu}^{\mu} \delta b^{\lambda} \dot{q}^{\nu}+2 S_{\lambda \nu}^{\mu} \dot{q}^{\lambda} \delta q^{\nu}
$$

Under an arbitrary nonholonomic variation $\delta q^{\mu}=\delta q^{\mu}+\delta b^{\mu}$, the action (3) changes by

$$
\delta \mathcal{A}=M \int_{t_{a}}^{t_{b}} d t\left(g_{\mu \nu} \dot{q}^{\nu} \delta \dot{q}^{\mu}+\frac{1}{2} \partial_{\mu} g_{\lambda \kappa} \delta q^{\mu} \dot{q}^{\lambda} \dot{q}^{\kappa}\right) .
$$

We use (67), (69) for a partial integration of the $\delta \dot{q}$-term, and apply the identity $\partial_{\mu} g_{\nu \lambda} \equiv$ $\Gamma_{\mu \nu \lambda}+\Gamma_{\mu \lambda \nu}$, which follows from the definitions $g_{\mu \nu} \equiv e^{i}{ }_{\mu} e^{i}{ }_{\nu}$ and $\Gamma_{\mu \nu}{ }^{\lambda} \equiv e_{i}{ }^{\lambda} \partial_{\mu} e^{i}{ }_{\nu}$, to obtain

$$
\delta \mathcal{A}=M \int_{t_{a}}^{t_{b}} d t\left[-g_{\mu \nu}\left(\ddot{q}^{\nu}+\bar{\Gamma}_{\lambda \kappa}{ }^{\nu} \dot{q}^{\lambda} \dot{q}^{\kappa}\right) \delta q^{\mu}+\left(g_{\mu \nu} \dot{q}^{\nu} \frac{d}{d t} \delta b^{\mu}+\Gamma_{\mu \lambda \kappa} \delta b^{\mu} \dot{q}^{\lambda} \dot{q}^{\kappa}\right)\right] .
$$

To derive the equation of motion we first vary the action in a space without torsion. Then $\delta b^{\mu}(t) \equiv 0$, and we obtain

$$
\delta \mathcal{A}=\delta \mathcal{A}=-M \int_{t_{a}}^{t_{b}} d t g_{\mu \nu}\left(\ddot{q}^{\nu}+\bar{\Gamma}_{\lambda \kappa}{ }^{\nu} \dot{q}^{\lambda} \dot{q}^{\kappa}\right) q^{\nu}
$$

Thus, the action principle $\delta \mathcal{A}=0$ produces the equation for the geodesics (9), which are the correct particle trajectories in the absence of torsion.

In the presence of torsion where $\delta b^{\mu} \neq 0$, the equation of motion receives a contribution from the second parentheses in (76). After inserting (74), the nonlocal terms proportional to $\delta b^{\mu}$ cancel and the total nonholonomic variation of the action becomes

$$
\begin{aligned}
\delta \mathcal{A} & =-M \int_{t_{a}}^{t_{b}} d t g_{\mu \nu}\left[\ddot{q}^{\nu}+\left(\bar{\Gamma}_{\lambda \kappa}^{\nu}+2 S^{\nu}{ }_{\lambda \kappa}\right) \dot{q}^{\lambda} \dot{q}^{\kappa}\right] \delta q^{\mu} \\
& =-M \int_{t_{a}}^{t_{b}} d t g_{\mu \nu}\left(\ddot{q}^{\nu}+\Gamma_{\lambda \kappa}{ }^{\nu} \dot{q}^{\lambda} \dot{q}^{\kappa}\right) \delta q^{\mu} .
\end{aligned}
$$

The second line follows from the first after using the identity $\Gamma_{\lambda \kappa}{ }^{\nu}=\bar{\Gamma}_{\{\lambda \kappa\}}{ }^{\nu}+2 S^{\nu}{ }_{\{\lambda \kappa\}}$. The curly brackets indicate the symmetrization of the enclosed indices. Setting $\delta \mathcal{A}=0$ gives the autoparallels (21) as the equations of motions, which is what we wanted to show.

In order appreciate the geometric significance of the differential equation (74), we introduce the matrices

$$
G^{\mu}(t)_{\lambda} \equiv \Gamma_{\lambda \nu}^{\mu}(q(t)) \dot{q}^{\nu}(t)
$$

and

$$
\Sigma_{\nu}^{\mu}(t) \equiv 2 S_{\lambda \nu}{ }^{\mu}(q(t)) \dot{q}^{\lambda}(t)
$$


and rewrite Eq. (74) as a differential equation for a vector

$$
\frac{d}{d t} \delta b=-G \delta b+\Sigma(t) \delta q^{\nu}(t)
$$

The solution is

$$
\delta b(t)=\int_{t_{a}}^{t} d t^{\prime} U\left(t, t^{\prime}\right) \Sigma\left(t^{\prime}\right) \delta q\left(t^{\prime}\right)
$$

with the matrix

$$
U\left(t, t^{\prime}\right)=T \exp \left[-\int_{t^{\prime}}^{t} d t^{\prime \prime} G\left(t^{\prime \prime}\right)\right] .
$$

In the absence of torsion, $\Sigma(t)$ vanishes identically and $\delta b(t) \equiv 0$, and the variations $\delta q^{\mu}(t)$ coincide with the holonomic $\delta q^{\mu}(t)$ [see Fig. 2(b)]. In a space with torsion, the variations $\delta q^{\mu}(t)$ and $\delta q^{\mu}(t)$ are different from each other [see Fig. 2(c)].

\subsection{Alternative Formulation of Action Principle with Torsion}

The above variational treatment of the action is still somewhat complicated and calls for a simpler procedure which was found recently [16].

Let us vary the paths $q^{\mu}(t)$ in the usual holonomic way, i.e., with fixed endpoints, and consider the associated variations $\delta x^{i}=e^{i}{ }_{\mu}(q) \delta q^{\mu}$ of the Cartesian coordinates. Taking their time derivative $d_{t} \equiv d / d t$ we find

$$
d_{t} \delta x^{i}=e_{\lambda}^{i}(q) d_{t} \delta q^{\lambda}+\partial_{\mu} e_{\lambda}^{i}(q) \dot{q}^{\mu} \delta q^{\lambda}
$$

On the other hand, we may write the relation (24) in the form $d_{t} x^{i}=e^{i}{ }_{\mu}(q) d_{t} q^{\mu}$ and vary this to yield

$$
\delta d_{t} x^{i}=e_{\lambda}^{i}(q) \delta \dot{q}^{\lambda}+\partial_{\mu} e_{\lambda}^{i}(q) \dot{q}^{\lambda} \delta q^{\mu} .
$$

Using now the fact that time derivatives $d_{t}$ and variations $\delta$ commute for Cartesian paths,

$$
\delta d_{t} x^{i}-d_{t} \delta x^{i}=0
$$

we deduce from (84) and (85) that this is no longer true in the presence of torsion, where

$$
\delta d_{t} q^{\lambda}-d_{t} \delta q^{\lambda}=2 S_{\mu \nu}^{\lambda}(q) \dot{q}^{\mu} \delta q^{\nu}
$$

In other words, the variations of the velocities $\dot{q}^{\mu}(t)$ no longer coincide with the time derivatives of the variations of $q^{\mu}(t)$.

This failure to commute is responsible for shifting the trajectory from geodesics to autoparallels. Indeed, let us vary an action

$$
\mathcal{A}=\int_{t_{a}}^{t_{b}} d t L\left(q^{\lambda}(t), \dot{q}^{\lambda}(t)\right)
$$


by $\delta q^{\lambda}(t)$ and impose (87), we find

$$
\delta \mathcal{A}=\int_{t_{a}}^{t_{b}} d t\left\{\frac{\partial L}{\partial q^{\lambda}} \delta q^{\lambda}+\frac{\partial L}{\partial \dot{q}^{\lambda}} \frac{d}{d t} \delta q^{\lambda}+2 S_{\mu \nu}^{\lambda} \frac{\partial L}{\partial \dot{q}^{\lambda}} \dot{q}^{\mu} \delta q^{\nu}\right\} .
$$

After a partial integration of the second term using the vanishing $\delta q^{\lambda}(t)$ at the endpoints, we obtain the Euler-Lagrange equation

$$
\frac{\partial L}{\partial q^{\lambda}}-\frac{d}{d t} \frac{\partial L}{\partial \dot{q}^{\lambda}}=2 S_{\lambda \mu}^{\nu} \dot{q}^{\mu} \frac{\partial L}{\partial \dot{q}^{\nu}}
$$

This differs from the standard Euler-Lagrange equation by an additional contribution due to the torsion tensor. For the action (3) we thus obtain the equation of motion

$$
M\left[\ddot{q}^{\lambda}+g^{\lambda \kappa}\left(\partial_{\mu} g_{\nu \kappa}-\frac{1}{2} \partial_{\kappa} g_{\mu \nu}\right)+2 S_{\mu \nu}^{\lambda}\right] \dot{q}^{\mu} \dot{q}^{\nu}=0
$$

which is once more Eq. (21) for autoparallels.

\section{Path Integral in Spaces with Curvature and Torsion}

We now turn to the quantum mechanics of a point particle in a general metric-affine space. We first consider the path integral in a flat space with Cartesian coordinates

$$
\left(\mathbf{x} t \mid \mathbf{x}^{\prime} t^{\prime}\right)=\frac{1}{\sqrt{2 \pi i \epsilon \hbar / M}} \prod_{n=1}^{N}\left[\int_{-\infty}^{\infty} d x_{n}\right] \prod_{n=1}^{N+1} K_{0}^{\epsilon}\left(\Delta \mathbf{x}_{n}\right)
$$

where $K_{0}^{\epsilon}\left(\Delta \mathbf{x}_{n}\right)$ is an abbreviation for the short-time amplitude

$$
K_{0}^{\epsilon}\left(\Delta \mathbf{x}_{n}\right) \equiv\left\langle\mathbf{x}_{n}\left|\exp \left(-\frac{i}{\hbar} \epsilon \hat{H}\right)\right| \mathbf{x}_{n-1}\right\rangle=\frac{1}{\sqrt{2 \pi i \epsilon \hbar / M}^{D}} \exp \left[\frac{i}{\hbar} \frac{M}{2} \frac{\left(\Delta \mathbf{x}_{n}\right)^{2}}{\epsilon}\right]
$$

with $\Delta \mathbf{x}_{n} \equiv \mathbf{x}_{n}-\mathbf{x}_{n-1}, \mathbf{x} \equiv \mathbf{x}_{N+1}, \mathbf{x}^{\prime} \equiv \mathbf{x}_{0}$. A possible external potential has been omitted since this would contribute in an additive way, uninfluenced by the space geometry.

Our basic postulate is that the path integral in a general metric-affine space should be obtained by an appropriate nonholonomic transformation of the amplitude (92) to a space with curvature and torsion.

\subsection{Nonholonomic Transformation of the Action}

The short-time action contains the square distance $\left(\Delta \mathbf{x}_{n}\right)^{2}$ which we have to transform to $q$-space. For an infinitesimal coordinate difference $\Delta \mathbf{x}_{n} \approx d \mathbf{x}_{n}$, the square distance 
is obviously given by $(d \mathbf{x})^{2}=g_{\mu \nu} d q^{\mu} d q^{\nu}$. For a finite $\Delta \mathbf{x}_{n}$, however, it is well known that we must expand $\left(\Delta \mathbf{x}_{n}\right)^{2}$ up to the fourth order in $\Delta q_{n}{ }^{\mu}=q_{n}{ }^{\mu}-q_{n-1}{ }^{\mu}$ to find all terms contributing to the relevant order $\epsilon$.

It is important to realize that with the mapping from $d x^{i}$ to $d q^{\mu}$ not being holonomic, the finite quantity $\Delta q^{\mu}$ is not uniquely determined by $\Delta x^{i}$. A unique relation can only be obtained by integrating the functional relation (65) along a specific path. The preferred path is the classical orbit, i.e., the autoparallel in the $q$-space. It is characterized by being the image of a straight line in the $x$-space. There $\dot{x}^{i}(t)=$ const and the orbit has the linear time dependence

$$
\Delta x^{i}(t)=\dot{x}^{i}\left(t_{0}\right) \Delta t
$$

where the time $t_{0}$ can lie anywhere on the $t$-axis. Let us choose for $t_{0}$ the final time in each interval $\left(t_{n}, t_{n-1}\right)$. At that time, $\dot{x}_{n}^{i} \equiv \dot{x}^{i}\left(t_{n}\right)$ is related to $\dot{q}_{n}^{\mu} \equiv \dot{q}^{\mu}\left(t_{n}\right)$ by

$$
\dot{x}_{n}^{i}=e_{\mu}^{i}\left(q_{n}\right) \dot{q}_{n}^{\mu} \text {. }
$$

It is easy to express $\dot{q}_{n}^{\mu}$ in terms of $\Delta q_{n}^{\mu}=q_{n}^{\mu}-q_{n-1}^{\mu}$ along the classical orbit. First we expand $q^{\mu}\left(t_{n-1}\right)$ into a Taylor series around $t_{n}$. Dropping the time arguments, for brevity, we have

$$
\Delta q \equiv q^{\lambda}-q^{\prime \lambda}=\epsilon \dot{q}^{\lambda}-\frac{\epsilon^{2}}{2 !} \ddot{q}^{\lambda}+\frac{\epsilon^{3}}{3 !} \dot{q}^{\lambda}+\ldots,
$$

where $\epsilon=t_{n}-t_{n-1}$ and $\dot{q}^{\lambda}, \ddot{q}^{\lambda}, \ldots$ are the time derivatives at the final time $t_{n}$. An expansion of this type is referred to as a postpoint expansion. Due to the arbitrariness of the choice of the time $t_{0}$ in Eq. (95), the expansion can be performed around any other point just as well, such as $t_{n-1}$ and $\bar{t}_{n}=\left(t_{n}+t_{n-1}\right) / 2$, giving rise to the so-called prepoint or midpoint expansions of $\Delta q$.

Now, the term $\ddot{q}^{\lambda}$ in (96) is given by the equation of motion (21) for the autoparallel

$$
\ddot{q}^{\lambda}=-\Gamma_{\mu \nu}{ }^{\lambda} \dot{q}^{\mu} \dot{q}^{\nu}
$$

A further time derivative determines

$$
\ddot{q}^{\lambda}=-\left(\partial_{\sigma} \Gamma_{\mu \nu}^{\lambda}-2 \Gamma_{\mu \nu}^{\tau} \Gamma_{\{\sigma \tau\}}^{\lambda}\right) \dot{q}^{\mu} \dot{q}^{\nu} \dot{q}^{\sigma}
$$

Inserting these expressions into (96) and inverting the expansion, we obtain $\dot{q}^{\lambda}$ at the final time $t_{n}$ expanded in powers of $\Delta q$. Using (94) and (95) we arrive at the mapping of the finite coordinate differences:

$$
\begin{aligned}
& \Delta x^{i}=e_{\lambda}^{i} \dot{q}^{\lambda} \Delta t \\
& \quad=e_{\lambda}^{i}\left[\Delta q^{\lambda}-\frac{1}{2 !} \Gamma_{\mu \nu}{ }^{\lambda} \Delta q^{\mu} \Delta q^{\nu}+\frac{1}{3 !}\left(\partial_{\sigma} \Gamma_{\mu \nu}{ }^{\lambda}+\Gamma_{\mu \nu}{ }^{\tau} \Gamma_{\{\sigma \tau\}}{ }^{\lambda}\right) \Delta q^{\mu} \Delta q^{\nu} \Delta q^{\sigma}+\ldots\right]
\end{aligned}
$$

where $e_{\lambda}^{i}$ and $\Gamma_{\mu \nu}{ }^{\lambda}$ are evaluated at the postpoint. Inserting this into the short-time amplitude (93), we obtain

$$
K_{0}^{\epsilon}(\Delta \mathbf{x})=\left\langle\mathbf{x}\left|\exp \left(-\frac{i}{\hbar} \epsilon \hat{H}\right)\right| \mathbf{x}-\Delta \mathbf{x}\right\rangle=\frac{1}{\sqrt{2 \pi i \epsilon \hbar / M}}{ }^{D} \exp \left[\frac{i}{\hbar} \mathcal{A}_{>}^{\epsilon}(q, q-\Delta q)\right]
$$


with the short-time postpoint action

$$
\begin{aligned}
\mathcal{A}_{>}^{\epsilon}( & q, q-\Delta q)=\frac{M}{2 \epsilon}\left(\Delta x^{i}\right)^{2}=\epsilon \frac{M}{2} g_{\mu \nu} \dot{q}^{\mu} \dot{q}^{\nu} \\
= & \frac{M}{2 \epsilon}\left\{g_{\mu \nu} \Delta q^{\mu} \Delta q^{\nu}-\Gamma_{\mu \nu \lambda} \Delta q^{\mu} \Delta q^{\nu} \Delta q^{\lambda}\right. \\
& \left.+\left[\frac{1}{3} g_{\mu \tau}\left(\partial_{\kappa} \Gamma_{\lambda \nu}{ }^{\tau}+\Gamma_{\lambda \nu}{ }^{\delta} \Gamma_{\{\kappa \delta\}}{ }^{\tau}\right)+\frac{1}{4} \Gamma_{\lambda \kappa}{ }^{\sigma} \Gamma_{\mu \nu \sigma}\right] \Delta q^{\mu} \Delta q^{\nu} \Delta q^{\lambda} \Delta q^{\kappa}+\ldots\right\} .
\end{aligned}
$$

Separating the affine connection into Christoffel symbol and torsion, this can also be written as

$$
\begin{aligned}
& \mathcal{A}_{>}^{\epsilon}(q, q-\Delta q)=\frac{M}{2 \epsilon}\left\{g_{\mu \nu} \Delta q^{\mu} \Delta q^{\nu}-\bar{\Gamma}_{\mu \nu \lambda} \Delta q^{\mu} \Delta q^{\nu} \Delta q^{\lambda}\right. \\
& \left.+\left[\frac{1}{3} g_{\mu \tau}\left(\partial_{\kappa} \bar{\Gamma}_{\lambda \nu}{ }^{\tau}+\bar{\Gamma}_{\lambda \nu}{ }^{\delta} \bar{\Gamma}_{\delta \kappa}{ }^{\tau}\right)+\frac{1}{4} \bar{\Gamma}_{\lambda \kappa}{ }^{\sigma} \bar{\Gamma}_{\mu \nu \sigma}+\frac{1}{3} S^{\sigma}{ }_{\lambda \kappa} S_{\sigma \mu \nu}+\ldots\right\}\right] \Delta q^{\mu} \Delta q^{\nu} \Delta q^{\lambda} \Delta q^{\kappa} .
\end{aligned}
$$

Note that the right-hand side contains only quantities intrinsic to the $q$-space. For the systems treated there (which all live in a euclidean space parametrized with curvilinear coordinates), the present intrinsic result reduces to the previous one.

At this point we observe that the final short-time action (101) could also have been introduced without any reference to the flat coordinates $x^{i}$. Indeed, the same action is obtained by evaluating the continuous action (3) for the small time interval $\Delta t=\epsilon$ along the classical orbit between the points $q_{n-1}$ and $q_{n}$. Due to the equations of motion (21), the Lagrangian

$$
L(q, \dot{q})=\frac{M}{2} g_{\mu \nu}(q(t)) \dot{q}^{\mu}(t) \dot{q}^{\nu}(t)
$$

is independent of time (this is true for autoparallels as well as geodesics). The short-time action

$$
\mathcal{A}^{\epsilon}\left(q, q^{\prime}\right)=\frac{M}{2} \int_{t-\epsilon}^{t} d t^{\prime} g_{\mu \nu}\left(q\left(t^{\prime}\right)\right) \dot{q}^{\mu}\left(t^{\prime}\right) \dot{q}^{\nu}\left(t^{\prime}\right)
$$

can therefore be written in either of the three forms

$$
\mathcal{A}^{\epsilon}=\frac{M}{2} \epsilon g_{\mu \nu}(q) \dot{q}^{\mu} \dot{q}^{\nu}=\frac{M}{2} \epsilon g_{\mu \nu}\left(q^{\prime}\right) \dot{q}^{\prime \mu} \dot{q}^{\prime \nu}=\frac{M}{2} \epsilon g_{\mu \nu}(\bar{q}) \dot{\bar{q}}^{\mu} \dot{\bar{q}}^{\nu}
$$

where $q^{\mu}, q^{\prime \mu}, \bar{q}^{\mu}$ are the coordinates at the final time $t_{n}$, the initial time $t_{n-1}$, and the average time $\left(t_{n}+t_{n-1}\right) / 2$, respectively. The first expression obviously coincides with (101). The others can be used as a starting point for deriving equivalent prepoint or midpoint actions. The prepoint action $\mathcal{A}_{<}^{\epsilon}$ arises from the postpoint one $\mathcal{A}_{>}^{\epsilon}$ by exchanging $\Delta q$ by $-\Delta q$ and the postpoint coefficients by the prepoint ones. The midpoint action has the most simple-looking appearance:

$$
\begin{aligned}
\overline{\mathcal{A}}^{\epsilon}(\bar{q}+ & \left.\frac{\Delta q}{2}, \bar{q}-\frac{\Delta q}{2}\right) \\
& =\frac{M}{2 \epsilon}\left[g_{\mu \nu}(\bar{q}) \Delta q^{\mu} \Delta q^{\nu}+\frac{1}{12} g_{\kappa \tau}\left(\partial_{\lambda} \Gamma_{\mu \nu}{ }^{\tau}+\Gamma_{\mu \nu}{ }^{\delta} \Gamma_{\{\lambda \delta\}}{ }^{\tau}\right) \Delta q^{\mu} \Delta q^{\nu} \Delta q^{\lambda} \Delta q^{\kappa}+\ldots\right]
\end{aligned}
$$


where the affine connection can be evaluated at any point in the interval $\left(t_{n-1}, t_{n}\right)$. The precise position is irrelevant to the amplitude producing only changes beyond the relevant order epsilon.

In the textbook [12], the postpoint action turned out to be the most useful one since it gives ready access to the time evolution of amplitudes. The prepoint action is completely equivalent to it and useful if one wants to describe the time evolution backwards. Some authors favor the midpoint action because of its symmetry and intimate relation to an ordering prescription in operator quantum mechanics which was advocated by $\mathrm{H}$. Weyl. This prescription is, however, only of historic interest since it does not lead to the correct physics. In the following, the action $\mathcal{A}^{\epsilon}$ without subscript will always denote the preferred postpoint expression (101):

$$
\mathcal{A}^{\epsilon} \equiv \mathcal{A}_{>}^{\epsilon}(q, q-\Delta q)
$$

\subsection{The Measure of Path Integration}

We now turn to the integration measure in the Cartesian path integral (92)

$$
\frac{1}{\sqrt{2 \pi i \epsilon \hbar / M}} \prod_{n=1}^{N} d^{D} x_{n}
$$

This has to be transformed to the general metric-affine space. We imagine evaluating the path integral starting out from the latest time and performing successively the integrations over $x_{N}, x_{N-1}, \ldots$, i.e., in each short-time amplitude we integrate over the earlier position coordinate, the prepoint coordinate. For the purpose of this discussion, we relabel the product $\prod_{n=1}^{N} d^{D} x_{n}^{i}$ by $\prod_{n=2}^{N+1} d x_{n-1}^{i}$, so that the integration in each time slice $\left(t_{n}, t_{n-1}\right)$ with $n=N+1, N, \ldots$ runs over $d x_{n-1}^{i}$.

In a flat space parametrized with curvilinear coordinates, the transformation of the integrals over $d^{D} x_{n-1}^{i}$ into those over $d^{D} q_{n-1}^{\mu}$ is obvious:

$$
\prod_{n=2}^{N+1} \int d^{D} x_{n-1}^{i}=\prod_{n=2}^{N+1}\left\{\int d^{D} q_{n-1}^{\mu} \operatorname{det}\left[e_{\mu}^{i}\left(q_{n-1}\right)\right]\right\} .
$$

The determinant of $e_{\mu}^{i}$ is the square root of the determinant of the metric $g_{\mu \nu}$ :

$$
\operatorname{det}\left(e_{\mu}^{i}\right)=\sqrt{\operatorname{det} g_{\mu \nu}(q)} \equiv \sqrt{g(q)}
$$

and the measure may be rewritten as

$$
\prod_{n=2}^{N+1} \int d^{D} x_{n-1}^{i}=\prod_{n=2}^{N+1}\left[\int d^{D} q_{n-1}^{\mu} \sqrt{g\left(q_{n-1}\right)}\right] .
$$

This expression is not directly applicable. When trying to do the $d^{D} q_{n-1}^{\mu}$-integrations successively, starting from the final integration over $d q_{N}^{\mu}$, the integration variable $q_{n-1}$ 
appears for each $n$ in the argument of $\operatorname{det}\left[e_{\mu}^{i}\left(q_{n-1}\right)\right]$ or $g_{\mu \nu}\left(q_{n-1}\right)$. To make this $q_{n-1^{-}}$ dependence explicit, we expand in the measure (108) $e_{\mu}^{i}\left(q_{n-1}\right)=e_{\mu}^{i}\left(q_{n}-\Delta q_{n}\right)$ around the postpoint $q_{n}$ into powers of $\Delta q_{n}$. This gives

$$
d x^{i}=e_{\mu}^{i}(q-\Delta q) d q^{\mu}=e_{\mu}^{i} d q^{\mu}-e_{\mu, \nu}^{i} d q^{\mu} \Delta q^{\nu}+\frac{1}{2} e_{\mu, \nu \lambda}^{i} d q^{\mu} \Delta q^{\nu} \Delta q^{\lambda}+\ldots
$$

omitting, as before, the subscripts of $q_{n}$ and $\Delta q_{n}$. Thus the Jacobian of the coordinate transformation from $d x^{i}$ to $d q^{\mu}$ is

$$
J_{0}=\operatorname{det}\left(e^{i}{ }_{\kappa}\right) \operatorname{det}\left[\delta^{\kappa}{ }_{\mu}-e_{i}{ }^{\kappa} e^{i}{ }_{\mu, \nu} \Delta q^{\nu}+\frac{1}{2} e_{i}{ }^{\kappa} e^{i}{ }_{\mu, \nu \lambda} \Delta q^{\nu} \Delta q^{\lambda}\right],
$$

giving the relation between the infinitesimal integration volumes $d^{D} x^{i}$ and $d^{D} q^{\mu}$ :

$$
\prod_{n=2}^{N+1} \int d^{D} x_{n-1}^{i}=\prod_{n=2}^{N+1}\left\{\int d^{D} q_{n-1}^{\mu} J_{0 n}\right\} .
$$

The well-known expansion formula

$$
\operatorname{det}(1+B)=\exp \operatorname{tr} \log (1+B)=\exp \operatorname{tr}\left(B-B^{2} / 2+B^{3} / 3-\ldots\right)
$$

allows us now to rewrite $J_{0}$ as

$$
J_{0}=\operatorname{det}\left(e^{i}{ }_{\kappa}\right) \exp \left(\frac{i}{\hbar} \mathcal{A}_{J_{0}}^{\epsilon}\right)
$$

with the determinant $\operatorname{det}\left(e_{\mu}^{i}\right)=\sqrt{g(q)}$ evaluated at the postpoint. This equation defines an effective action associated with the Jacobian, for which we obtain the expansion

$$
\frac{i}{\hbar} \mathcal{A}_{J_{0}}^{\epsilon}=-e_{i}{ }^{\kappa} e^{i}{ }_{\kappa, \mu} \Delta q^{\mu}+\frac{1}{2}\left[e_{i}{ }^{\mu} e_{\mu, \nu \lambda}^{i}-e_{i}{ }^{\mu} e^{i}{ }_{\kappa, \nu} e_{j}{ }^{\kappa} e^{j}{ }_{\mu, \lambda}\right] \Delta q^{\nu} \Delta q^{\lambda}+\ldots
$$

To express this in terms of the affine connection, we use (19) and derive the relations

$$
\begin{aligned}
\frac{1}{4} e_{i \nu, \mu} e^{i}{ }_{\kappa, \lambda} & =\frac{1}{4} e_{i}{ }^{\sigma} e^{i}{ }_{\nu, \mu} e_{j \sigma} e^{j}{ }_{\kappa, \lambda}=\frac{1}{4} \Gamma_{\mu \nu}{ }^{\sigma}, \Gamma_{\lambda \kappa \sigma} \\
\frac{1}{3} e_{i \mu} e^{i}{ }_{\nu, \lambda \kappa} & =\frac{1}{3} g_{\mu \tau}\left[\partial_{\kappa}\left(e_{i}{ }^{\tau} e^{i}{ }_{\nu, \lambda}\right)-e^{i \sigma} e_{\nu, \lambda}^{i} e^{j}{ }_{\sigma} e^{j \tau}{ }_{, \kappa}\right] \\
& =\frac{1}{3} g_{\mu \tau}\left(\partial_{\kappa} \Gamma_{\lambda \nu}{ }^{\tau}+\Gamma_{\lambda \nu}{ }^{\sigma} \Gamma_{\kappa \sigma}{ }^{\tau}\right)
\end{aligned}
$$

With these, the Jacobian action becomes

$$
\frac{i}{\hbar} \mathcal{A}_{J_{0}}^{\epsilon}=-\Gamma_{\mu \nu}{ }^{\nu} \Delta q^{\mu}+\frac{1}{2} \partial_{\mu} \Gamma_{\nu \kappa}{ }^{\kappa} \Delta q^{\nu} \Delta q^{\mu}+\ldots
$$

The same result would, of course, be obtained by writing the Jacobian in accordance with $(110)$ as

$$
J_{0}=\sqrt{g(q-\Delta q)}
$$


which leads to the alternative formula for the Jacobian action

$$
\exp \left(\frac{i}{\hbar} \mathcal{A}_{J_{0}}^{\epsilon}\right)=\frac{\sqrt{g(q-\Delta q)}}{\sqrt{g(q)}}
$$

An expansion in powers of $\Delta q$ gives

$$
\exp \left(\frac{i}{\hbar} \mathcal{A}_{\bar{J}_{0}}^{\epsilon}\right)=1-\frac{1}{\sqrt{g(q)}} \sqrt{g(q)}{ }_{, \mu} \Delta q^{\mu}+\frac{1}{2 \sqrt{g(q)}} \sqrt{g(q)}{ }_{, \mu \nu} \Delta q^{\mu} \Delta q^{\nu}+\ldots
$$

Using the formula

$$
\frac{1}{\sqrt{g}} \partial_{\mu} \sqrt{g}=\frac{1}{2} g^{\sigma \tau} \partial_{\mu} g_{\sigma \tau}=\bar{\Gamma}_{\mu \nu}{ }^{\nu}
$$

this becomes

$$
\exp \left(\frac{i}{\hbar} \mathcal{A}_{\bar{J}_{0}}^{\epsilon}\right)=1-\bar{\Gamma}_{\mu \nu}{ }^{\nu} \Delta q^{\mu}+\frac{1}{2}\left(\partial_{\mu} \bar{\Gamma}_{\nu \lambda}{ }^{\lambda}+\bar{\Gamma}_{\mu \sigma}{ }^{\sigma} \bar{\Gamma}_{\nu \lambda}{ }^{\lambda}\right) \Delta q^{\mu} \Delta q^{\nu}+\ldots
$$

so that

$$
\frac{i}{\hbar} \mathcal{A}_{\bar{J}_{0}}^{\epsilon}=-\bar{\Gamma}_{\mu \nu}{ }^{\nu} \Delta q^{\mu}+\frac{1}{2} \partial_{\mu} \bar{\Gamma}_{\nu \lambda}{ }^{\lambda} \Delta q^{\mu} \Delta q^{\nu}+\ldots
$$

In a space without torsion where $\bar{\Gamma}_{\mu \nu}^{\lambda} \equiv \Gamma_{\mu \nu}^{\lambda}$, the Jacobian actions (119) and (125) are trivially equal to each other. But the equality holds also in the presence of torsion. Indeed, when inserting the decomposition (29), $\Gamma_{\mu \nu}{ }^{\lambda}=\bar{\Gamma}_{\mu \nu}{ }^{\lambda}+K_{\mu \nu}{ }^{\lambda}$, into (119), the contortion tensor drops out since it is antisymmetric in the last two indices and these are contracted in both expressions. form

In terms of $\mathcal{A}_{J_{0 n}}^{\epsilon}$, we can rewrite the transformed measure (108) in the more useful

$$
\prod_{n=2}^{N+1} \int d^{D} x_{n-1}^{i}=\prod_{n=2}^{N+1}\left\{\int d^{D} q_{n-1}^{\mu} \operatorname{det}\left[e_{\mu}^{i}\left(q_{n}\right)\right] \exp \left(\frac{i}{\hbar} \mathcal{A}_{J_{0 n}}^{\epsilon}\right)\right\} .
$$

In a flat space parametrized in terms of curvilinear coordinates, the right-hand sides of (108) and (126) are related by an ordinary coordinate transformation, and both give the correct measure for a time-sliced path integral. In a general metric-affine space, however, this is no longer true. Since the mapping $d x^{i} \rightarrow d q^{\mu}$ is nonholonomic, there are in principle infinitely many ways of transforming the path integral measure from Cartesian coordinates to a noneuclidean space. Among these, there exists a preferred mapping which leads to the correct quantum-mechanical amplitude in all known physical systems. It is this mapping which led to the correct solution of the path integral of the hydrogen atom [11].

The clue for finding the correct mapping is offered by an unesthetic feature of Eq. (111): The expansion contains both differentials $d q^{\mu}$ and differences $\Delta q^{\mu}$. This 
is somehow inconsistent. When time-slicing the path integral, the differentials $d q^{\mu}$ in the action are increased to finite differences $\Delta q^{\mu}$. Consequently, the differentials in the measure should also become differences. A relation such as (111) containing simultaneously differences and differentials should not occur.

It is easy to achieve this goal by changing the starting point of the nonholonomic mapping and rewriting the initial flat space path integral (92) as

$$
\left(\mathbf{x} t \mid \mathbf{x}^{\prime} t^{\prime}\right)=\frac{1}{\sqrt{2 \pi i \epsilon \hbar / M}} \prod_{n=1}^{N}\left[\int_{-\infty}^{\infty} d \Delta x_{n}\right] \prod_{n=1}^{N+1} K_{0}^{\epsilon}\left(\Delta \mathbf{x}_{n}\right)
$$

Since $x_{n}$ are Cartesian coordinates, the measures of integration in the time-sliced expressions (92) and (127) are certainly identical:

$$
\prod_{n=1}^{N} d^{D} x_{n} \equiv \prod_{n=2}^{N+1} d^{D} \Delta x_{n}
$$

Their images under a nonholonomic mapping, however, are different so that the initial form of the time-sliced path integral is a matter of choice. The initial form (127) has the obvious advantage that the integration variables are precisely the quantities $\Delta x_{n}^{i}$ which occur in the short-time amplitude $K_{0}^{\epsilon}\left(\Delta x_{n}\right)$.

Under a nonholonomic transformation, the right-hand side of Eq. (128) leads to the integral measure in a general metric-affine space

$$
\prod_{n=2}^{N+1} \int d^{D} \Delta x_{n} \rightarrow \prod_{n=2}^{N+1}\left[\int d^{D} \Delta q_{n} J_{n}\right]
$$

with the Jacobian following from (99) (omitting $n$ )

$$
\begin{aligned}
J & =\frac{\partial(\Delta x)}{\partial(\Delta q)} \\
& =\operatorname{det}\left(e^{i}{ }_{\kappa}\right) \operatorname{det}\left[\delta_{\mu}{ }^{\lambda}-\Gamma_{\{\mu \nu\}}{ }^{\lambda} \Delta q^{\nu}+\frac{1}{2}\left(\partial_{\sigma} \Gamma_{\mu \nu}{ }^{\lambda}+\Gamma_{\{\mu \nu}{ }^{\tau} \Gamma_{\{\tau \mid \sigma\}\}}{ }^{\lambda}\right) \Delta q^{\nu} \Delta q^{\sigma}+\ldots\right] .
\end{aligned}
$$

In a space with curvature and torsion, the measure on the right-hand side of (129) replaces the flat-space measure on the right-hand side of (110). The curly double brackets around the indices $\nu, \kappa, \sigma, \mu$ indicate a symmetrization in $\tau$ and $\sigma$ followed by a symmetrization in $\mu, \nu$, and $\sigma$. With the help of formula (114) we now calculate the Jacobian action

$$
\begin{aligned}
\frac{i}{\hbar} \mathcal{A}_{J}^{\epsilon=} & -\Gamma_{\{\mu \nu\}}^{\mu} \Delta q^{\nu} \\
& +\frac{1}{2}\left[\partial_{\{\mu} \Gamma_{\nu \kappa\}}{ }^{\kappa}+\Gamma_{\{\nu \kappa}{ }^{\sigma} \Gamma_{\{\sigma \mid \mu\}\}}{ }^{\kappa}-\Gamma_{\{\nu \kappa\}}{ }^{\sigma} \Gamma_{\{\sigma \mu\}}{ }^{\kappa}\right] \Delta q^{\nu} \Delta q^{\mu}+\ldots .
\end{aligned}
$$

The curly double brackets around the indices $\nu, \kappa, \sigma, \mu$ indicate a symmetrization in $\tau$ and $\sigma$ followed by a symmetrization in $\mu, \nu$, and $\sigma$ (here the index $\mu$ is excluded as 
indicated by the bar). This expression differs from the earlier Jacobian action (119) by the symmetrization symbols. Dropping them, the two expressions coincide. This is allowed if $q^{\mu}$ are curvilinear coordinates in a flat space. Since then the transformation functions $x^{i}(q)$ and their first derivatives $\partial_{\mu} x^{i}(q)$ are integrable and possess commuting derivatives, the two Jacobian actions (119) and (131) are identical.

There is a further good reason for choosing (128) as a starting point for the nonholonomic transformation of the measure. According to Huygens' principle of wave optics, each point of a wave front is a center of a new spherical wave propagating from that point. Therefore, in a time-sliced path integral, the differences $\Delta x_{n}^{i}$ play a more fundamental role than the coordinates themselves. Intimately related to this is the observation that in the canonical form, a short-time piece of the action reads

$$
\int \frac{d p_{n}}{2 \pi \hbar} \exp \left[\frac{i}{\hbar} p_{n}\left(x_{n}-x_{n-1}\right)-\frac{i p_{n}^{2}}{2 M \hbar} t\right] .
$$

Each momentum is associated with a coordinate difference $\Delta x_{n} \equiv x_{n}-x_{n-1}$. Thus, we should expect the spatial integrations conjugate to $p_{n}$ to run over the coordinate differences $\Delta x_{n}=x_{n}-x_{n-1}$ rather than the coordinates $x_{n}$ themselves, which makes the important difference in the subsequent nonholonomic coordinate transformation.

We are thus led to postulate the following time-sliced path integral in $q$-space:

$$
\begin{aligned}
\left\langle q\left|\exp \left[-\frac{i}{\hbar}\left(t-t^{\prime}\right) \hat{H}\right]\right| q^{\prime}\right\rangle= & \frac{1}{\sqrt{2 \pi i \hbar \epsilon / M}} \prod_{n=2}^{N+1}\left[\int d^{D} \Delta q_{n} \frac{\sqrt{g\left(q_{n}\right)}}{\sqrt{2 \pi i \epsilon \hbar / M}}\right] \\
& \times \exp \left[\frac{i}{\hbar} \sum_{n=1}^{N+1}\left(\mathcal{A}^{\epsilon}+\mathcal{A}_{J}^{\epsilon}\right)\right],
\end{aligned}
$$

where the integrals over $\Delta q_{n}$ may be performed successively from $n=N$ down to $n=1$.

Let us emphasize that this expression has not been derived from the flat space path integral. It is the result of a specific new quantum equivalence principle which rules how a flat space path integral behaves under nonholonomic coordinate transformations.

It is useful to reexpress our result in a different form which clarifies best the relation with the naively expected measure of path integration (110), the product of integrals

$$
\prod_{n=1}^{N} \int d^{D} x_{n}=\prod_{n=1}^{N}\left[\int d^{D} q_{n} \sqrt{g\left(q_{n}\right)}\right] .
$$

The measure in (133) can be expressed in terms of (134) as

$$
\prod_{n=2}^{N+1}\left[\int d^{D} \Delta q_{n} \sqrt{g\left(q_{n}\right)}\right]=\prod_{n=1}^{N}\left[\int d^{D} q_{n} \sqrt{g\left(q_{n}\right)} e^{-i \mathcal{A}_{J_{0}}^{\epsilon} / \hbar}\right] .
$$

The corresponding expression for the entire time-sliced path integral (133) in the metricaffine space reads

$$
\left\langle q\left|\exp \left[-\frac{i}{\hbar}\left(t-t^{\prime}\right) \hat{H}\right]\right| q^{\prime}\right\rangle=\frac{1}{\sqrt{2 \pi i \hbar \epsilon / M}^{D}} \prod_{n=1}^{N}\left[\int d^{D} q_{n} \frac{\sqrt{g\left(q_{n}\right)}}{\sqrt{2 \pi i \hbar \epsilon / M}^{D}}\right]
$$




$$
\times \exp \left[\frac{i}{\hbar} \sum_{n=1}^{N+1}\left(\mathcal{A}^{\epsilon}+\Delta \mathcal{A}_{J}^{\epsilon}\right)\right]
$$

where $\Delta \mathcal{A}_{J}^{\epsilon}$ is the difference between the correct and the wrong Jacobian actions in Eqs. (119) and (131):

$$
\Delta \mathcal{A}_{J}^{\epsilon} \equiv \mathcal{A}_{J}^{\epsilon}-\mathcal{A}_{J_{0}}^{\epsilon}
$$

In the absence of torsion where $\Gamma_{\{\mu \nu\}}^{\lambda}=\bar{\Gamma}_{\mu \nu}{ }^{\lambda}$, this simplifies to

$$
\frac{i}{\hbar} \Delta \mathcal{A}_{J}^{\epsilon}=\frac{1}{6} \bar{R}_{\mu \nu} \Delta q^{\mu} \Delta q^{\nu}
$$

where $\bar{R}_{\mu \nu}$ is the Ricci tensor associated with the Riemann curvature tensor, i.e., the contraction (40) of the Riemann curvature tensor associated with the Christoffel symbol $\bar{\Gamma}_{\mu \nu}{ }^{\lambda}$.

Being quadratic in $\Delta q$, the effect of the additional action can easily be evaluated perturbatively using the methods explained in Chapter 8 of the textbook [12], according to which $\Delta q^{\mu} \Delta q^{\nu}$ may be replaced by its lowest order expectation

$$
\left\langle\Delta q^{\mu} \Delta q^{\nu}\right\rangle_{0}=i \epsilon \hbar g^{\mu \nu}(q) / M
$$

Then $\Delta \mathcal{A}_{J}^{\epsilon}$ yields the additional effective potential

$$
V_{\text {eff }}=-\frac{\hbar^{2}}{6 M} \bar{R}
$$

where $\bar{R}$ is the Riemann curvature scalar. 2 By including this potential in the action, the path integral in a curved space can be written down in the naive form (134) as follows:

$$
\begin{aligned}
& \left\langle q\left|\exp \left[-\frac{i}{\hbar}\left(t-t^{\prime}\right) \hat{H}\right]\right| q^{\prime}\right\rangle=\frac{1}{\sqrt{2 \pi i \hbar \epsilon / M}} \prod_{n=1}^{N}\left[\int d^{D} q_{n} \frac{\sqrt{g\left(q_{n}\right)}}{\sqrt{2 \pi i \epsilon \hbar / M}}\right] \\
& \times \exp \left[\frac{i}{\hbar} \sum_{n=1}^{N+1}\left(\mathcal{A}^{\epsilon}+\epsilon V_{\text {eff }}\right)\right] \text {. }
\end{aligned}
$$

The integrals over $q_{n}$ are conveniently performed successively downwards over $\Delta q_{n+1}=$ $q_{n+1}-q_{n}$ at fixed $q_{n+1}$. The weights $\sqrt{g\left(q_{n}\right)}=\sqrt{g\left(q_{n+1}-\Delta q_{n+1}\right)}$ require a postpoint expansion leading to the naive Jacobian $J_{0}$ of (112) and the Jacobian action $\mathcal{A}_{J_{0}}^{\epsilon}$ of Eq. (119).

\footnotetext{
${ }^{2}$ This is one of the $\bar{R}$-terms of DeWitt. Another term with opposite sign and a factor $-1 / 2$ was found by him from the prefactor in the DeWitt-Morette semiclassical amplitude which he employed for the short-time propagator. See the discussion in Appendix 11B of [12].
} 
It goes without saying that the path integral (139) also has a phase space version. It is obtained by omitting all $(M / 2 \epsilon)\left(\Delta q_{n}\right)^{2}$ terms in the short-time actions $\mathcal{A}^{\epsilon}$ and extending the multiple integral by the product of momentum integrals

$$
\prod_{n=1}^{N+1}\left[\frac{d p_{n}}{2 \pi \hbar \sqrt{g\left(q_{n}\right)}}\right] e^{(i / \hbar) \sum_{n=1}^{N+1}\left[p_{n \mu} \Delta q^{\mu}-\epsilon \frac{1}{2 M} g^{\mu \nu}\left(q_{n}\right) p_{n \mu} p_{n \nu}\right]} .
$$

When using this expression, all problems which were encountered in the literature with canonical transformations of path integrals disappear.

\section{Conclusion}

It appears as though the new variational and quantum equivalence principles constitute the proper basis for a correct extension of our physical laws into geometries with torsion. In both principles, nonholonomic mappings play a fundamental role. When applied to classical paths, these mappings lead directly to the new variational principle and thus to the correct equations of motion. Their correctness is a consequence of the fact that classical equations of motion remain valid under nonholonomic coordinate transformations. An important application not discussed here is the derivation of the Euler-Lagrange equations of a spinning top within the rotating body-fixed frame of references from an extremum of the kinetic action [18].

The quantum equivalence principle adds to the nonholonomic mapping procedure the postulate that the measure of path integration which is to be mapped into a space with curvature and torsion contains the same time-sliced intervals $\Delta x^{i}$ which appear in the short-time action [see Eq. (127)]. The most important theoretical evidence for the correctness of this principle comes from the solution of the path integral of the Coulomb problem. This was presented in the Lectures, but will not be repeated here, referring the reader to the textbook [12]. Only with this measure has it been possible to find the solution without undesirable time-slicing corrections.

Another theoretical evidence which was mentioned only briefly in the lectures comes from the bosonization of Fermi theories [19-28]. Only with the new measure is this bosonization possible [29] without errors in the energy spectrum.

\section{References}

[1] B.S. DeWitt, Rev. Mod. Phys. 29, 377 (1957).

[2] B. Podolsky, Phys. Rev. 32, 812 (1928).

[3] K.S. Cheng, J. Math. Phys. 13, 1723 (1972);

H. Kamo and T. Kawai, Prog. Theor. Phys. 50, 680 (1973);

T. Kawai, Found. Phys. 5, 143 (1975);

H. Dekker, Physica A 103, 586 (1980); 
G.M. Gavazzi, Nuovo Cimento A 101, 241 (1981).

A good survey over similar attempts is given by

M.S. Marinov, Phys. Rep. 60, 1 (1980).

[4] Among the most widely discussed procedures was a postpoint discretization due to Ito and a midpoint discretization due to Stratonovich, with different mathematical advantages. For a detailed discussion see the textbooks

H. Risken, The Fokker-Planck Equation, second edition, Springer, 1983, Vol. 18;

R. Kubo, M. Toda, and N. Hashitsume, Statistical Physics II, Springer, Berlin 1985.

A recent description of the relation between time slicing and Ito versus Stratonovich calculus can be found in

H. Nakazato, K. Okano, L. Schülke, and Y. Yamanaka, Nucl. Phys. B 346, 611 (1990).

Stochastic differential equations in curved spaces are developed in

K.D. Elworthy, Stochastic differential equations on manifolds, Cambridge Univ. Press, 1982;

M. Emery, Stochastic calculus in manifolds, Springer, Berlin 1989.

[5] R. Graham, Z. Phys. B 26, 397 (1977).

[6] K.D. Elworthy, Path Integration on Manifolds, in Mathematical Aspects of Superspace, eds. H.-J. Seifert, C. Clarke, and A. Rosenblum, Reidel, 1984.

[7] L.D. Landau and E.M. Lifshitz, Quantum Mechanics, Pergamon, New York, 1965.

[8] D.J. Simms and N. M. J. Woodhouse, Lectures on geometric quantization, Springer, Berlin 1976;

J. Sniatycki, Geometric quantization and quantum mechanics, Springer, Berlin 1980;

P.L. Robinson and J.H. Rawnsley, The metaplectic representation, Mpc structures, and geometric quantization, publ. by the American Mathematical Society in the series Memoirs of the American Mathematical Society no. 410 0065-9266, Providence, R.I., 1989.

[9] For details and more references see H. Kleinert, Gauge Fields in Condensed Matter, Vol. I Superflow and Vortex Lines, pp. 1-744, and Vol. II Stresses and Defects, World Scientific, Singapore 1989, pp. 744-1443.

[10] K. Kondo, in: Proc. 2nd Japan Nat. Congr. Applied Mechanics, Tokio, 1952 B.A. Bilby, R. Bullough and E. Smith, Proc. R. Soc. London A 231, 263 (1955); E. Kröner, in: Physics of defects, Les Houches summer school XXXV, NorthHolland, Amsterdam 1981.

[11] H. Duru and H. Kleinert, Phys. Lett. B 84, 185 (1979); Fortschr. d. Phys. 30, 401 (1982). 
[12] H. Kleinert, Path Integrals in Quantum Mechanics, Statistics and Polymer Physics,, second edition, World Scientific, Singapore 1995.

[13] H. Kleinert, Mod. Phys. Lett. A 4, 2329 (1989).

[14] H. Kleinert, Phys. Lett. B 236, 315 (1990).

[15] P. Fiziev and H. Kleinert, New Action Principle for Classical Particle Trajectories In Spaces with Torsion, Europh. Lett. 35, 2411996 (hep-th/9503074 and http://www.physik.fu-berlin.de/ kleinert/kleiner_re219/newvar.htm]).

[16] A. Pelster and H. Kleinert, FU-Berlin preprint, May 1996 (gr-qc/9605028 and http://www.physik.fu-berlin.de/ kleinert/kleiner_re243/preprint.html).

[17] Our notation for the geometric quantities in spaces with curvature and torsion is the same as in

J.A. Schouten, Ricci Calculus, Springer, Berlin 1954.

[18] P. Fiziev and H. Kleinert, Euler Equations for Rigid-Body - A Case for Autoparallel Trajectories in Spaces with Torsion, Berlin preprint 1995 (hep-th/9503075 and http://www.physik.fu-berlin.de/〜kleinert/kleiner_re224/euler.html).

[19] H. Kleinert, Collective Quantum Fields, Lectures presented at the First Erice Summer School on Low-Temperature Physics, 1977, Fortschr. Physik 26, 565-671 (1978).

See also the predecessors:

H. Kleinert, Field Theory of Collective Excitations - A Soluble Model, Phys. Lett. B 69, 9 (1977),

as well as the derivation of an $\mathrm{SU}(3) \times \mathrm{SU}(3)$ chirally invariant field theory of mesons from a quark theory in

H. Kleinert, Hadronization of Quark Theories and a Bilocal form of QED, Phys. Lett. B 62, 429 (1976);

H. Kleinert, On the Hadronization of Quark Theories, Lectures presented at the Erice Summer Institute 1976, in

Understanding the Fundamental Constituents of Matter,

Plenum Press, New York, 1978, A. Zichichi ed., pp. 289-390.

[20] L.P. Gorkov, Sov. Phys. JETP 9, 1364 (1959).

[21] V.L. Ginzburg and L.D. Landau, Eksp. Teor. Fiz. 20, 1064 (1950).

[22] A.L. Leggett, Rev. Mod. Phys. 47, 331 (1975).

[23] K.D. Schotte and U. Schotte, Phys. Rev. 182, 479 (1969);

see also:

S. Tomonaga, Progr. Theor. Phys. 5, 63 (1950). 
[24] For a review see:

D.R. Bes, R.A. Broglia, Lectures delivered at "E. Fermi" Varenna Summer School, Varenna, Como Italy, 1976. For recent studies: D.R. Bes, R.A. Broglia, R. Liotta, B.R. Mottelson, Phys. Letters B 52, 253 (1974); B 56, 109 (1975), Nuclear Phys. A 260, 127 (1976).

See also:

R.W. Richardson, J. Math. Phys. 9, 1329 (1968), Ann. Phys. (N.Y.) 65, 249 (1971) and N.Y.U. Preprint 1977, as well as references therein.

[25] J. Hubbard, Phys. Rev. Letters 3, 77 (1959); B. Mühlschlegel, J. Math. Phys. , 3, 522 (1962); J. Langer, Phys. Rev. A 134, 553 (1964); T.M. Rice, Phys. Rev. A 1401889 (1965); J. Math. Phys. 8, 1581 (1967); A.V. Svidzinskij, Teor. Mat. Fiz. 9, 273 (1971); D. Sherrington, J. Phys. C 4, 401 (1971).

[26] E. Witten, Commun. Math. Phys. 92, 455 (1984);

P. DiVecchia and P. Rossi, Phys. Lett. B 140, 344 (1984);

P. DiVecchia, B. Durhuus and J.L. Petersen, Phys. Lett. B 144, 245 (1984);

Y. Frishman, Phys. Lett. B 146, 204 (1984);

E. Abdalla and M.C.B. Abdalla, Nucl. Phys. B 225, 392 (1985);

D. Gonzales and A.N. Redlich, Phys. Lett. B 147, 150 (1984);

C.M. Naón, Phys. Rev. D 31, 2035 (1985);

See also the recent development by

P.H. Damgaard, H.B. Nielsen, and R. Sollacher, Nuclear Phys. B 385, 227 (1992) (hep-th/9407022);

P.H. Damgaard and R. Sollacher, Cern preprint (hep-th/9407022);

A.N. Theron; F.A. Schaposnik, F. G. Scholtz and H.B. Geyer, Nucl. Phys. B 437, 187 (1995) (hep-th/9410035);

C.P. Burgess and F. Quevedo, Phys. Lett. B 329 (1994) 457; Nucl. Phys. B 421, 373 (1994);

C.P. Burgess, A. Lutkin, and F. Quevedo, Phys. Lett. B 336, 18 (1994);

J. Fröhlich, R. Götschmann and P.A. Marchetti, preprint (hep-th/9406154).

[27] S. Coleman, Phys. Rev. D 11, 2088 (1975);

S. Mandelstam, Phys. Rev. D 11, 3026 (1975);

B. Schroer and T.T. Truong, Phys. Rev. D 15, 1684 (1977).

[28] For a semiclassical study of the model at finite times see

H. Kleinert and H. Reinhardt, Nucl. Phys. A 332, 33 (1979).

[29] H. Kleinert, Nonabelian Bosonization as a Nonholonomic Transformations from Flat to Curved Field Space. FU-Berlin preprint 1996 (http://www.physik.fuberlin.de/^kleinert/kleiner_re239/preprint.htm]). 


\section{FIGURES}

Fig. 1: Crystal with dislo dinate transformations from introduces torsion and no $\mathrm{cl}$

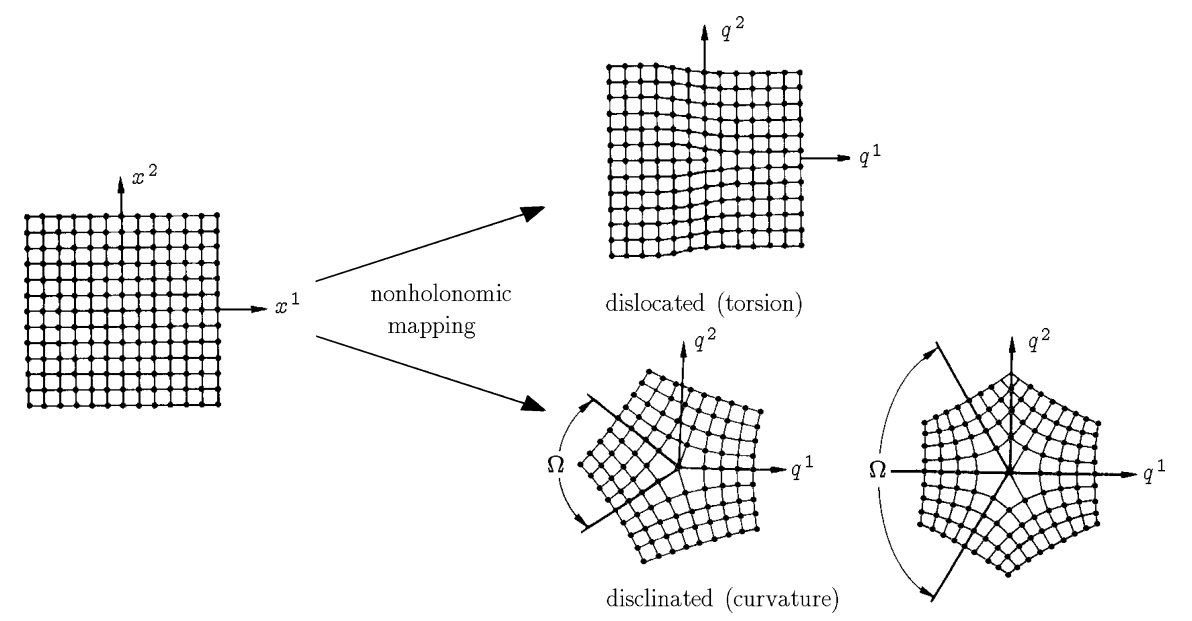

Fig. 2: Images under a holonomic and a nonholonomic mapping of a fundamental path variation. In the holonomic case, the paths $x(t)$ and $x(t)+\delta x(t)$ in (a) turn into the paths $q(t)$ and $q(t)+\delta q(t)$ in (b). In the nonholonomic case with $S_{\mu \nu}{ }^{\lambda} \neq 0$, they go over into $q(t)$ and $q(t)+\delta q(t)$ shown in (c) with a closure failure $b^{\mu}$ at $t_{b}$ analogous to the Burgers vector $b^{\mu}$ in a solid with dislocations.

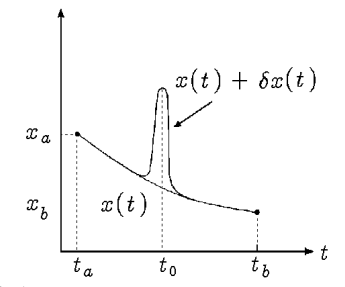

(a)

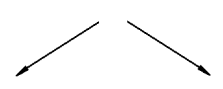

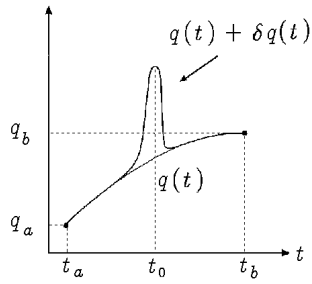

(b)

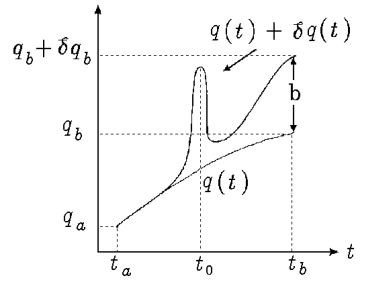

(c) 